\title{
Effect on outcome of the lengthening waiting list for liver transplantation
}

Liver transplantation is now recognised as a highly effective form of treatment for patients with end stage liver disease. Currently, one and five year survival rates are in excess of $80 \%$. As a consequence of many developments in all aspects of transplantation, indications have widened and contraindications become fewer. However, the increase in the number of patients referred for transplantation has not been matched by an increase in donor rates. Despite the innovative use of techniques to make fullest use of the donor pool, such as splitting of livers and increasing use of marginal livers (those livers where there is doubt as to initial graft function), the demand for transplantation is failing to meet the supply. The consequence of this relative shortage of donors is that more patients are on the waiting list for transplantation, those on the waiting list become sicker before they receive a graft, and there is the likelihood of more deaths on the waiting list.

In the UK the majority of patients are referred by gastroenterologists in district general hospitals to one of the seven designated liver transplant units. To make the most efficient use of these scarce livers, it is important that those clinicians referring patients for transplantation are aware of the problems consequent upon the donor shortage.

Selection of patients for liver transplantation occurs at three stages. The first stage is when the patient's doctor (usually a gastroenterologist working in a district general hospital) refers the patient for transplantation. We believe that the greatest selection occurs at this point. The second stage is when the patient is referred to the transplant unit, is assessed, and accepted on the transplant list. Indications for liver transplantation and contraindications are now relatively well established ${ }^{1}$ so it is not surprising that most referred patients are accepted onto the list; in our unit, about one fifth of patients are not accepted either because it is considered too early for listing or, less commonly, because there are contraindications to transplantation. In the USA, where early referral is more common than in the UK, minimal listing criteria have been introduced. ${ }^{2}$ The third stage of selection occurs when a donor liver becomes available. Selection of the optimal recipient is dependent on many factors: some factors such as matching the donor and recipient for size and blood group are major determinants of selection. Other factors depend on the relative health of the patient and quality of the liver. For example, it may be inappropriate to use a marginal liver in a very sick patient as they would be unlikely to tolerate the early postoperative period.

Understanding those factors which are associated with the risk of dying prior to transplantation may allow improvements in referral for transplantation and improvement in the management of the waiting list.

\section{Situation in the UK}

The gap between the number of patients listed for transplantation and number of donor organs is widening in the UK, continental Europe, and North America. ${ }^{3}$ Data for transplantation in the UK are shown in table 1 . While there was an increase in the number of transplants performed between 1990 and 1997, the rate of increase has been slow in the past few years and there remains a discrepancy between the number of transplants performed and number of new patients registered. In the UK, during the first nine months of 1999 the active liver transplant list increased by $12 \%$ compared with the same period in 1998 (from 178 to 199) whereas the number of transplants (whole liver and lobe) from cadaveric donors increased by only $2 \%$ (from 436 to 445 ). During this period, there was a $2 \%$ fall in the total number of cadaveric donors. There has been an increase in the number of patients dying while on the active transplant list and an increase in the number of patients removed from this list. The reasons for removal from the list are not clear but include patients becoming too ill to benefit from the procedure and temporary removal for intercurrent problems.

\section{Mortality on the waiting list}

To understand the causes of death and also to identify markers which could determine patients at high risk of dying on the waiting list, we undertook a retrospective analysis of all adult patients listed for transplantation at this centre between January 1990 and December 1996. Those with fulminant hepatic failure and multiple or repeat grafts were excluded. Overall, 726 patients were accepted for transplantation. By March 1997, 9\% of the patients had died on the list, $87 \%$ had been transplanted, and $3 \%$ remained on the list. The proportion of patients dying on the list each year varied between $5 \%$ and $12 \%$ with no significant variation between years. Furthermore, there was no correlation between the risk of dying on the waiting list and the overall list size. When considered by indications for transplantation, of the 254 patients listed for transplantation for primary biliary cirrhosis (PBC), 14 (5\%) died whereas 12 of 88 patients (14\%) with alcoholic liver disease (ALD) died. Of those patients who died awaiting transplantation, there was no significant difference in Child-Pugh score for those with PBC (median 11, range $7-14$ ) and ALD (median 13, range $11-14 ; \mathrm{p}=0.15$ ). Death was due to hepatocellular failure rather than a specific precipitating factor such as variceal bleed (14\%). It should be noted that many of these deaths from variceal haemorrhage occurred before the widespread introduction of routine prophylaxis with beta blocker therapy.

A multivariate analysis of clinical and biochemical factors was performed for patients with ALD and PBC. For patients with ALD, a low serum albumin and high

Table 1 Waiting list data for liver transplantation in the UK (data supplied by UKTSSA)

\begin{tabular}{lllllllll}
\hline & 1990 & 1991 & 1992 & 1993 & 1994 & 1995 & 1996 & 1997 \\
\hline New patients registered & 360 & 439 & 504 & 577 & 629 & 675 & 872 & 696 \\
Transplants & 358 & 421 & 508 & 534 & 620 & 669 & 640 & 671 \\
Died on active list & 37 & 30 & 42 & 57 & 51 & 56 & 59 & 66 \\
Removed from active list & 19 & 38 & 35 & 41 & 40 & 41 & 47 & 81 \\
\hline
\end{tabular}

Abbreviations used in this paper: PBC, primary biliary cirrhosis; ALD, alcoholic liver disease. 
serum bilirubin were associated with death on the waiting list. For patients with PBC, serum bilirubin alone correlated with mortality. There was no difference in waiting times to transplantation or death between those listed for PBC or ALD (median 62 days for PBC and 46 days for patients with $\operatorname{ALD}(p=0.2)$; for those who died awaiting a liver the median time from listing to death was 42 days for patients with ALD and 34 days for patients with PBC $(p=0.79))$. Of the other factors considered, including patient age, blood group, serum creatinine, clotting profile, Child-Pugh score, presence of ascites, or encephalopathy, none was associated with death on the waiting list. Overall, only high serum bilirubin and low serum albumin were associated with a risk of dying.

\section{Consequences for survival on waiting times for patients with primary biliary cirrhosis}

To understand further the effect of the waiting list on the mortality of patients awaiting transplantation and the outcome after transplantation, we studied in greater detail patients with PBC accepted for transplantation. This indication was chosen not only because it represents a significant indication for transplantation but also because prognostic models for assessment of survival with and without transplantation are well developed and validated. ${ }^{5}$ Of 387 patients accepted for transplantation, 21 died awaiting transplantation. Of those who were transplanted, the median interval between listing and transplantation was 46 days (25th and 75 th centiles 15 and 102 days). Clinical, laboratory, and prognostic parameters on admission and at listing are shown in table 2. Although there were statistically significant differences in important prognostic variables, these were clinically of little importance. We estimated at three time points the probability of six month survival with and without transplantation: at the time of referral, at listing, and at transplantation (table 3). For patients who were transplanted, it can be seen that there was relatively little difference in estimated outcome after transplantation at any of the three times although there was a decrease in the probability of survival without transplantation. When those patients who waited more than 90 days between listing and transplantation were compared with those who waited less than 90 days, it was found that both the probabilities of surviving after transplantation and without transplantation were higher at all times in those who waited longer for a graft. The probability of six month survival with transplantation at referral, listing, and transplantation was $92 \%, 92 \%$, and $93 \%$ for those who waited more than 90 days and $86 \%, 85 \%$, and $85 \%$ for those who waited less than 90 days, compared with the probability of surviving six months without transplantation of $93 \%, 91 \%$, and $90 \%$ and $48 \%, 29 \%$, and

Table 2 Characteristics of all patients with primary biliary cirrhosis at referral and at listing (values are medians (upper and lower quartiles))

\begin{tabular}{lrlrl}
\hline & \multicolumn{2}{c}{ Admission } & \multicolumn{2}{c}{ Listing } \\
\hline Bilirubin $(\mu \mathrm{mol} / \mathrm{l})$ & 135 & $(65-252)$ & 164 & $(82-289)$ \\
Albumin $(\mathrm{g} / \mathrm{l})$ & 30 & $(25-35)$ & 30 & $(26-34)$ \\
Creatinine $(\mu \mathrm{mol} / \mathrm{l})$ & 78 & $(67-91)$ & 80 & $(69-95)$ \\
Urea $(\mathrm{mmol} / \mathrm{l})$ & $4.5(3.4-6.2)$ & $4.7(3.6-6.5)$ \\
\hline
\end{tabular}

Table 3 Percent six month survival probabilities, with and without transplantation, for patients referred for transplantation who were transplanted. Survival probabilities are shown as medians (upper and lower quartiles)

\begin{tabular}{lll}
\hline & With transplantation & Without transplantation \\
\hline Referral & $88(81-93)$ & $86(60-94)$ \\
Listing & $88(81-92)$ & $83(48-93)$ \\
Transplantation & $88(79-92)$ & $79(36-92)$ \\
\hline
\end{tabular}

Table 4 Percent six month survival probability at referral and listing of patients with primary biliary cirrhosis who died before a graft became available

\begin{tabular}{llr}
\hline & With transplantation & Without transplantation \\
\hline Referral & $79(64-85)$ & $39(1-67)$ \\
Listing & $70(62-79)$ & $6(1-45)$ \\
\hline
\end{tabular}

$19 \%$, respectively. These values suggest that even though the models were not used in assessing patients, those more likely to die without transplantation waited a shorter period.

When those patients who died while awaiting a transplant were assessed (table 4), it can be seen that while the six month survival probability after transplantation was good, it was not as high as those who received a transplant; the estimated survival in the absence of transplantation was much lower at both referral and listing. One quarter of patients with PBC who died awaiting a graft had a serum bilirubin level greater than $575 \mu \mathrm{mol} / 1$ at referral; in contrast, of those who were transplanted, the top quartile had, at referral, serum bilirubin levels above $252 \mu \mathrm{mol} / 1$.

While these findings are reassuring and suggest that the waiting time did not adversely impact on the outcome of most patients, the mortality awaiting a liver is of major concern. Some caution must be applied to the findings: models are useful at predicting population survival but are less so when applied to individuals. Confidence intervals are wide. Finally, these findings are based on a retrospective analysis.

\section{Conclusions}

Inevitably this analysis is limited by the retrospective nature of this study. None the less, several conclusions are apparent. Firstly, mortality on the waiting list is a significant problem and is likely to rise further until donor shortage can be reversed or alternative strategies can be introduced. Secondly, timing of referral for consideration of transplantation remains problematic. If patients are referred and listed early for transplantation then, because of the perioperative risk and small early mortality, there is a risk the patient's life might be shortened. Early and preemptive listing of patients to allow for the increased waiting time results in unmanageable waiting lists, as evidenced in North America; whether the introduction of minimal listing criteria will alter the practice remains to be seen. Conversely, referral too late not only reduces the survival of patients if they are transplanted but increases mortality on the waiting list. As shown here and elsewhere, ${ }^{6}$ those factors that predict survival after transplantation differ from those which predict survival without transplantation. Mortality of patients on the waiting list relates primarily to end stage disease rather than sudden and unexpected complications such as variceal haemorrhage or infection. Those patients who die on the list are usually not on the waiting list for long periods of time and have a slightly lower survival probability after transplantation. The transplant clinicians need to establish guidelines to determine when the patient's chances of survival are so low that transplantation becomes no longer indicated. A better understanding by the transplant clinicians of those factors which predict outcome with and without transplantation may help in improving the management of the waiting list; these data suggest that, at least for patients with PBC, priority for transplantation should be given to those who are most likely to die as this will not have a major adverse impact on survival after transplantation. For the referring clinician, early referral is important. 
It remains essential that transplant centres and referring clinicians work closely so that each clinical group understands the problems and stresses of the other thus ensuring that patient referral is optimal.

We are grateful to Mrs Robina Balderson and Dr Philip Pocock for supplying information from the UKTSSA

R RANSFORD

B GUNSON

D MAYER

J NEUBERGER

Liver Unit, Queen Elizabeth Hospital, Birmingham B15 2TH, UK

E CHRISTENSEN

Clinic of Internal Medicine I, Bispebjerg University Hospital, Copenhagen, Denmark
Correspondence to: Professor J Neuberger. Email: j.m.neuberger@bham.ac.uk

1 Willaims R, Portmann B, Tan KC, eds. The practice of liver transplantation. Edinburgh: Churchill Livingstone, 1995.

2 Lucey MR, Brown KA, Everson GT, et al. Minimal criteria for placement on the liver transplant waiting list. Transplantation 1998;66:95662 .

3 UNOS Scientific Registry, 1997. Report of the OPTN: waiting list activity and donor procurement. Richmond, Virginia: US Department of Health and Human Services, 1998.

4 Persijn GG, DeMeester JM. Demand, supply and allocation in Eurotransplant. Ann Transplant 1997;2:26-33.

5 Christensen E, Gunson B, Neuberger J. Optimal timing of liver transplantation for patients with primary biliary cirrhosis: use of prognostic modelling. tion for patients with prima

6 Ricci P, Therneau TM, Maclinchoc M, et al. A prognostic model for the outcome of liver transplantation in patients with cholestatic disease. Hepatology 1997;25:672-7. 